\title{
Potencial dendrocronológico de Jacaranda copaia que contiene registro ENSO en Madre de Dios, Perú
}

\author{
Leif Armando PORTAL Cahuana ${ }^{1 *}$, Jorge CARDOZO Soarez ${ }^{1}$, Lucio Ubaldo SANTOS Huacac ${ }^{1}$, \\ Gorky SAAVEDRA Alvarado², Bertín Alexis HUAMÁN Guevara ${ }^{1}$ \\ ${ }^{1}$ Universidad Nacional Amazónica de Madre de Dios, Puerto Maldonado, Perú. \\ (Orcid: *; 0000-0002-4653-7191; 0000-0003-0033-2024; 0000-0002-7505-2863) \\ ${ }^{2}$ Gobierno Regional de Madre de Dios, Puerto Maldonado, Perú. (Orcid: 0000-0002-3385-5449) \\ *E-mail: armantigre@gmail.com (Orcid: 0000-0002-2717-4348)
}

Recebido em 02/04/2020; Aceito em 26/08/2020; Publicado em 31/08/2020.

\begin{abstract}
RESUMEN: El objetivo de esta investigación fue construir la cronología de los anillos de crecimiento de la especie Jacaranda copaia (Achihua), analizar con los eventos más severos del fenómeno El Niño. Para ello se colectaron 10 árboles de J. copaia, de un bosque relicto de la región de Madre de Dios en el Perú. Se obtuvieron cuatro muestras por árbol, mediante la sonda de Pressler. El proceso, medición y análisis de los anillos de crecimiento fueron realizados mediante técnicas estándar de dendrocronología. Se construyó la cronología master de J. copaia, se determinó el incremento medio anual de la especie y la cronología fue relacionada con los eventos severos del fenómeno de El Niño. Los resultados muestran que J. copaia, presentan anillos de crecimiento distinto y anuales, delimitados por zonas fibrosas. La intercorrelación del ancho de los anillos de crecimiento entre árboles fue de 0,36. La edad máxima fue de 34 años. La relación entre la cronología y los eventos más severos del fenómeno El Niño fue de 0,18 y tuvo una relación clara en el año 1998. Los resultados revelan el potencial dendrocronológico de la especie J. copaia, en los bosques tropicales de Madre de Dios y para futuros estudios dendroclimáticos.
\end{abstract}

Palabras-clave: especie forestal; El Niño; anillos de crecimientos.

\section{Dendrochronology of Jacaranda copaia containing ENSO registry in Madre de Dios, Peru}

\begin{abstract}
The objective of this investigation was to construct the chronology of the growth rings of the Jacaranda copaia (Achihua) species, to analyze with the most severe events of the El Niño phenomenon. For this, $10 \mathrm{~J}$. copaia trees were collected from a relict forest in the Madre de Dios region of Peru. Four samples per tree were obtained using the Pressler's probe. The process, measurement and analysis of the growth rings were carried out using standard dendrochronology techniques. The J. copaia master chronology was constructed, the mean annual increase of the species was determined and the chronology was related to the severe events of the El Niño phenomenon. The results show that J. copaia, present distinct and annual growth rings, delimited by fibrous zones. The intercorrelation of the width of the growth rings between trees was 0,36 . The maximum age was 34 years. The relationship between the chronology and the most severe events of the El Niño phenomenon was 0,18 and had a clear relationship in 1998. The results reveal the dendrochronological potential of the $J$. copaia species, in the tropical forests of Madre de Dios in for future dendroclimatic studies.
\end{abstract}

Keywords: forest species; El Niño; growth rings.

\section{INTRODUCCIÓN}

Madre de Dios, ubicado en el sur oriente del Perú, es uno de los bosques tropicales, documentado por tener una gran diversidad flora y fauna con muchas especies endémicas, considerado como un reservorio de las riquezas biológicas del Perú (FOSTER; PARKER; GENTRY, 1994).

Por otra parte, el fenómeno de El Niño, también llamado Oscilación del Sur (ENSO) es el clima interanual natural más fuerte. El ENSO se origina en el océano tropical del Pacífico y tiene grandes efectos en la ecología de la región, pero también influye en todo el mundo. Puede entenderse como una oscilación irregular de baja frecuencia cálida (El Niño) y fría (La Niña). El fuerte fenómeno de El Niño, en las últimas décadas han estado marcadas por fuertes eventos, principalmente en: 1982/1983 y 1997/1998. También, se han realizado modelos climáticos globales donde están prediciendo aumentos en la frecuencia y magnitud del fenómeno El Niño, que se sabe que influye en la variabilidad interanual del clima de América del Sur. Los eventos de El Niño llevan a condiciones secas anómalas sobre la región amazónica (FELFILI et al., 2018; SCHONGART et al., 2004; TIMMERMANN et al., 1999).

Además, en América del Sur, diversas investigaciones se han realizado, a través de técnicas dendrocronológicas, relacionando el crecimiento de los árboles tropicales con la influencia del fenómeno El Niño. Estas investigaciones han demostrado que existe influencia del ENSO sobre el crecimiento de los árboles tropicales en América del Sur (BRIENEN, R. et al., 2012; BRIENEN, R.W.; ZUIDEMA, 2005; FELFILI et al., 2018; SCHONGART et al., 2004).

En los reportes de anillos de crecimiento anuales de los árboles tropicales, se incluye a las especies de la familia 
botánica Bignoniaceae (Tabla 1), principalmente especies de los géneros Gormania, Handroanthus, Jacaranda, Tabebuia y Zeyheria; en el género Jacaranda se tiene dos especies: Jacaranda copaia y J. cuspidifolia. Sin embargo, pocos estudios se han realizado con la especie J. copaia, desde estudios que comprobaron la anualidad de los anillos de crecimiento (DÉTIENNE, 1989) hasta los últimos años donde comprobaron el crecimiento y la reproducción de J. copaia, responden de manera diferente al clima en Panamá (ALFARO et al., 2017).

En este artículo, utilizamos técnicas dendrocronológicas a través de los anillos de crecimiento de J. copaia, una especie caducifolia, exigente a la luz, para determinar el potencial dendrocronológico de la especie en esta región del Perú, ¿cuál es su incremento radial? y si los eventos más severos del fenómeno El Niño está registrado en la cronología de la especie.

Tabla 1. Lista de especies de la familia Bignoniaceae, que presentan anillos de crecimiento anuales comprobados.

Table 1. List of species in the family Bignoniaceae, which have proven annual growth rings.

\begin{tabular}{|c|c|c|}
\hline Familia & Especies & Referencia \\
\hline \multirow{18}{*}{ 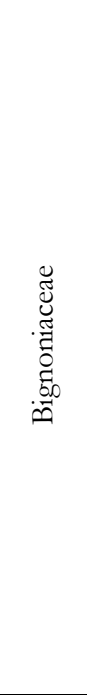 } & Godmania aesculifolia & \multirow{18}{*}{$\begin{array}{l}\text { (ANDRADE et al., 2019; } \\
\text { BELTRÁN; VALENCIA, 2013; } \\
\text { CALLADO et al., 2001; GARCÍA; } \\
\text { CAMARERO; ESPINOSA, 2017; } \\
\text { HAYDEN, 2009; LISI et al., 2008; } \\
\text { MENDIVELSO et al., 2014; } \\
\text { MENDIVELSO; CAMARERO; } \\
\text { GUTIÉRREZ, 2016; } \\
\text { SCHÖNGART et al., 2017; } \\
\text { VIEIRA; GROENENDIJK; LISI, } \\
\text { 2019; VOLLAND et al., 2011; } \\
\text { WORBES; RASCHKE, 2012) }\end{array}$} \\
\hline & Handroanthus barbatus & \\
\hline & H. chrysantbus & \\
\hline & H. impetiginosus & \\
\hline & H. serratifolius & \\
\hline & Jacaranda copaia & \\
\hline & J. cuspidifolia & \\
\hline & Tabebuia aurea & \\
\hline & T. billbergii & \\
\hline & T. cassinoides & \\
\hline & T. chrysantha & \\
\hline & T. chrysantha & \\
\hline & 1. heptaphylla & \\
\hline & $\frac{\text { 1. impetiginosa }}{\text { T. ochracea }}$ & \\
\hline & T. ocbracea & \\
\hline & T. rosea & \\
\hline & T. umbellata & \\
\hline & Zeyheria tuberculosa & \\
\hline
\end{tabular}

\section{MATERIAL Y MÉTODOS}

\section{1. Área de Estudio}

La colecta de muestras se realizó en el fundo El Bosque, de la Universidad Nacional Amazónica de Madre de Dios, ubicado en la carretera Interoceánica Sur kilómetro 16,5, margen derecho, dirección Puerto Maldonado-Iñapari, en el departamento de Madre de Dios en el Perú (Figura 02). Este departamento, es conocido por sus altos niveles de biodiversidad, denominada como capital de la biodiversidad del Perú en el año 1994 (Ley N²6311) hasta la actualidad (FOSTER; PARKER; GENTRY, 1994). El fundo, cuenta con aproximadamente con 460 hectáreas, de bosque relicto, que se clasifica como bosque de Terraza Alta. Es un bosque tropical húmedo, que se encuentra en promedio a una elevación de $250 \mathrm{msnm}$, presenta una temperatura media anual de $25^{\circ} \mathrm{C}$, la precipitación anual promedio es de 2200 a $2400 \mathrm{~mm}$ y en la época seca (julio a setiembre) (Figura 1), la precipitación promedio llega menos de $100 \mathrm{~mm}$ (MALHI et al., 2002).

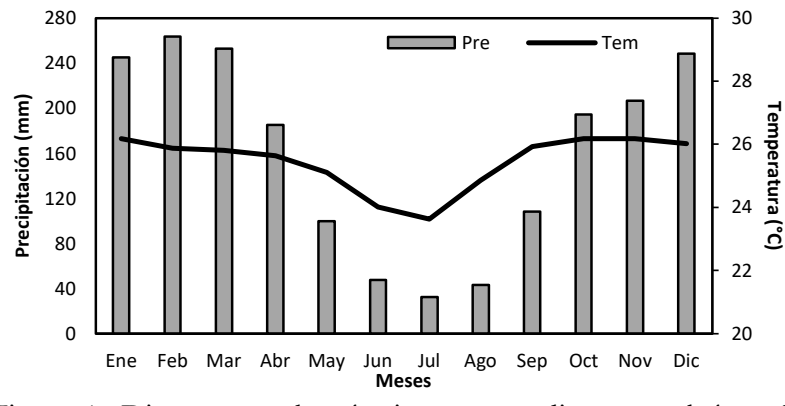

Figura 1. Diagrama ombrotérmico con medias para el área de estudio. (PORTAL, 2017).

Figure 1. Ombothermic diagram with means for the study area. (PORTAL, 2017).

\subsection{Inventario de los árboles, colección y extracción de las muestras}

Se realizó el inventario de la especie J. copaia, en el área de 5 hectáreas, recabando información dasométrica de circunferencia a la altura del pecho, diámetro a la altura del pecho, altura comercial, altura total, observaciones y coordenadas (Figura 2). De los 55 árboles inventariados, se seleccionaron al azar 10 árboles de J. copaia, con buen porte, estado fitosanitario bueno, tronco recto, de los cuales se extrajo 04 muestras de madera en dirección norte, sur, este y oeste.

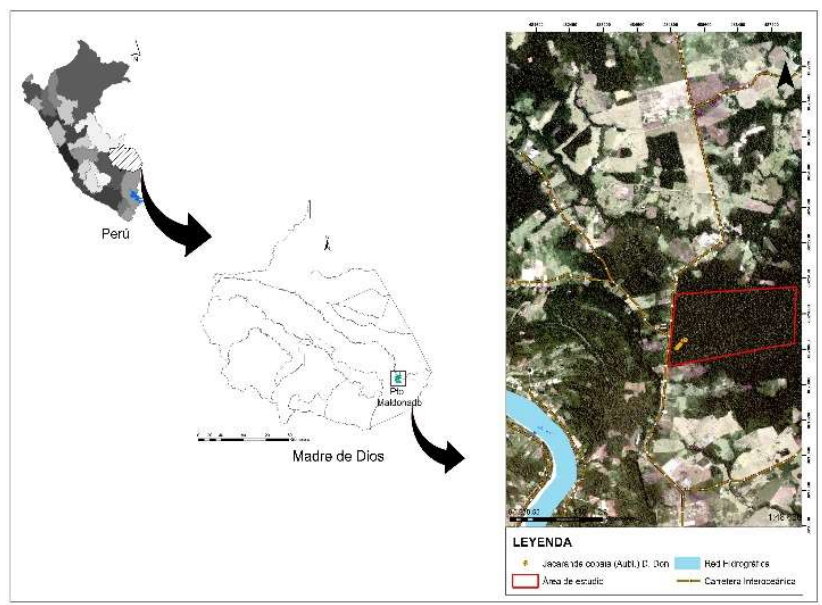

Figura 2. Mapa de ubicación del área de estudio, donde se muestra la ubicación de los árboles de J. copaia.

Figure 2. Location map of the study area, showing the location of the J. copaia trees.

Las muestras fueron colectadas con ayuda del instrumento no destructivo del Barreno o Sonda de Pressler, que tuvo una dimensión de 5,1 de diámetro x $400 \mathrm{~mm}$ de largo. Se obtuvieron cuatro muestras (radios) por árbol, en dirección corteza - médula, distanciadas a $90^{\circ}$ y colectadas al nivel del Diámetro a la Altura del Pecho (DAP), del fuste de los árboles seleccionados. Las muestras de leño, fueron acondicionados en tubos de plásticos, las cuales fueron codificadas para su fácil distinción y diferenciación entre los árboles y finalmente estas fueron trasladados al Laboratorio de Anatomía de la Madera de la Universidad Nacional Amazónica de Madre de Dios, ubicada en la Planta Piloto de Tecnología de la Madera - UNAMAD. 


\subsection{Preparación de las muestras de madera}

Las muestras de madera, obtenidas de los 10 árboles, fueron pegados en un soporte de madera y ajustadas con pabilo, considerando importante la sección transversal mirando hacia arriba. Los códigos de campo fueron respetados en cada soporte de madera. Posteriormente las series fueron secadas a temperatura ambiente y pulidas en una secuencia de lijas (60-1200 grano $\left./ \mathrm{cm}^{2}\right)$, para que se destaquen los anillos de crecimiento de J. copaia, en la sección transversal de las series.

\subsection{Identificación, caracterización, digitalización de las} muestras y medición de los anillos de crecimiento

La identificación de los anillos de crecimiento de los árboles de J. copaia, se realizó, observando la sección transversal con lupa de 10x y con microscopio estereoscopio Leica, marcando en el soporte de madera la delimitación de cada anillo de crecimiento. La Caracterización de los anillos de crecimiento se realizó en base a International Association of Wood Anatomists Committee (IAWA, 1989).

Asimismo, se digitalizaron las muestras de J. copaia, con un scanner a una resolución de 1200 dpi, con la finalidad de destacar los anillos y para que sea mejor su observación y posterior medición. Las imágenes digitalizadas de los árboles se midieron con el Software Image Pro Plus v 4.5, previo a este paso las escalas fueron calibradas. Luego de la medición del ancho de los anillos de crecimiento de manera manual, se exportaron los valores obtenidos por cada árbol hacia Miscrosoft Office Excel para ser ordenados y posteriormente analizados.

\subsection{Sincronización de los anillos de crecimiento y elaboración de la cronología}

El crossdating o sincronización cruzada, consistió en padronizar el ancho de los anillos de crecimiento dentro de cada árbol y entre árboles de J. copaia, permitió atribuir un año calendario para cada anillo de crecimiento identificado y medido.

Los datos del ancho de los anillos de crecimiento fueron procesados por el programa estadístico COFECHA versión 6.00p (HOLMES, 1983), para el control y verificación de la sincronización de las series entre y dentro de cada árbol; el programa además permite identificar la existencia de falsos anillos de crecimiento o anillos faltantes para un control posterior, además de permitir comprobar y corregir el sincronizado de las series.

Para la obtención de las cronologías de las especies, en el fundo El Bosque, se aplicó el software ARSTAN (MRWE Application Framework Copyright (C) 1997-2004) (HOLMES, 1983). El software permitió realizar la estandarización de las series individuales de cada árbol.

\subsection{Incremento radial}

Para realizar esta evaluación se utilizó 10 árboles seleccionados de J. copaia, se procedió a la determinación del crecimiento en diámetro del tronco de los árboles a partir de: 1) Incremento corriente anual en diámetro (ICAD). 2) Incremento diametral acumulado (IDA). 3) Incremento medio anual en diámetro (IMAD).

$$
\begin{aligned}
& \text { ICAD }=2 \times \text { Ancho del anillo } \\
& \mathrm{IDA}=\sum_{1}^{\mathrm{n}} \mathrm{ICAD}
\end{aligned}
$$

Posteriormente conocido el ICA para cada año, se calculó el incremento medio anual (IMA) para cada año de crecimiento, expresado como la sumatoria del incremento corriente anual:

$$
\mathrm{IMA}=\frac{\sum \mathrm{ICA} A_{\mathrm{DAP}}}{\mathrm{Edad}}
$$

\subsection{Cronología de Jacaranda copaia y relación con el clima y} el fenómeno El Niño.

La cronología de la especie J. copaia, fue correlacionadas con los datos históricos del fenómeno de El Niño, obtenido de la base del National Oceanic and Atmopheric Administration (NCEP-NCAR Reanalysis); esta correlación se realizó en cada trimestre (KALNAY et al. 1996; NOAA 2017).

\section{RESULTADOS}

\subsection{Caracterización de la sección transversal}

La sección transversal se caracteriza por presentar albura y duramen no diferenciado, de color crema blanquecina. Presenta una textura media. Poros: la porosidad es de tipo difusa, los poros son visibles a simple vista, poros mayormente solitarios y escasamente múltiples de 2 y 3 poros. Inclusiones ausentes. Parénquima: Es visible con la lupa de 10X, del tipo paratraqueal aliforme y confluente de alas delgadas. Radios: Son visibles con lupa de 10X, medianos (Figura3/B). Olor y sabor no distintivo.

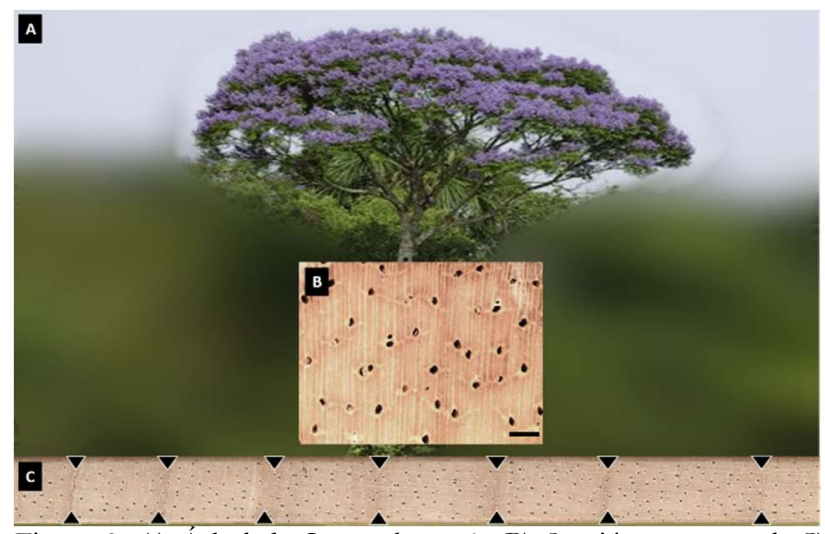

Figura 3. A) Árbol de Jacaranda copaia. B) Sección transversal. C) Límites de los anillos de crecimiento, delimitados por zonas fibrosas. Límites de los anillos indicados con fechas negras.

Figure 3. A) Jacaranda copaia tree. B) Cross section. C) Limits of the growth rings, delimited by fibrous areas. Ring boundaries indicated with black dates.

\subsection{Caracterización de los anillos de crecimiento}

La especie de J. copaia, en la sección transversal, se caracterizan anatómicamente por evidenciar anillos de crecimientos distintos a simple vista. El límite de los anillos de crecimiento es por zonas fibrosas de una coloración crema oscura (Figura 3/C). En la zona cerca a la médula, los anillos son poco diferencia o suavemente marcados. Sobre los anillos de crecimiento falsos, se caracterizan por ser discontinuos, de coloración tenue en comparación a los anillos verdaderos y son muy escasos.

\subsection{Dendrocronología}

Las series del ancho de los anillos de crecimiento de $J$. copaia (Figura 4), construida con muestras colectadas no destructivas en octubre del 2019, muestran en el ancho de sus 
Portal et al.

anillos de crecimiento tendencias similares de los 10 árboles de bosque natural.

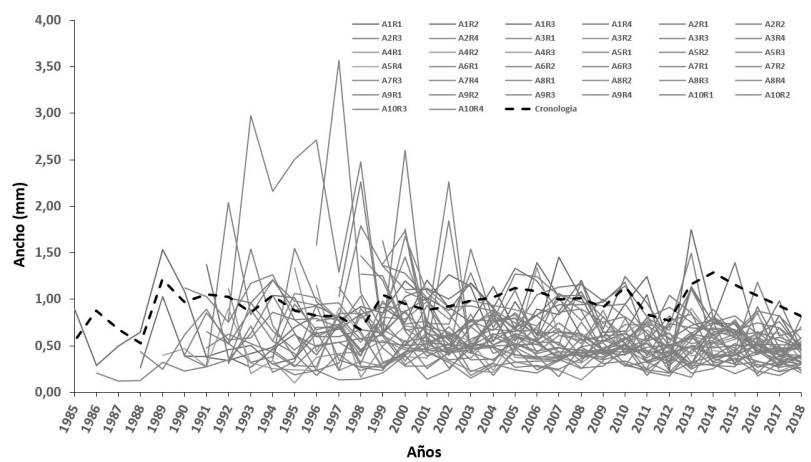

Figura 4. Anchos de los anillos de crecimiento de la especie J. copaia. Figure 4. Growth ring widths of the species J. copaia.

Se realizó el control de calidad de las medidas del ancho de los anillos de crecimiento de J. copaia, descartando 06 muestras radiales y manteniendo los 10 árboles colectados disponibles para sincronizar sus series cronológicas (Tabla 2).

Fue posible sincronizar las series de los anchos de los anillos de crecimientos de los 10 árboles de J. copaia, mediante el programa COFECHA. Los resultados muestran una cronología construida desde 1984 hasta el 2018, teniendo árboles más viejos de J. copaia, de 34 años y los más jóvenes de 19 años. Estos árboles de J. copaia, presentaron una intercorrelación de 0,361 para los 10 árboles, en promedio presento una sensibilidad de 0,367 (Tabla 3).

Luego de haber obtenido una intercorrelación mayor a 0,32 que establece el programa COFECHA, se utilizó el programa ARSTAN, para conseguir la cronología master de los árboles de J. copaia del fundo El Bosque de la UNAMAD. Dicha cronología master fue generada a partir de los 10 árboles colectados con 34 muestras radiales, construyendo una serie master de 34 años (1984-2018) (Figura 5).

\subsection{Incremento radial}

El crecimiento radial de los 10 árboles de la especie J.coapia, mostraron una variación en el ancho las camadas de crecimiento, siendo el árbol más joven de 19 años y el más viejo de 34 años (Figura 6). El diámetro a la altura del pecho "DAP" de los 10 árboles de J. copaia, vario de 19 a $39 \mathrm{~cm}$. Los 10 árboles de J. copaia, presentaron un similar patrón de crecimiento, siendo estos árboles de diferentes edades (Figura 6). El incremento medio anual para los 10 árboles promedio, mínimo y máximo fue de $0,80,0,30$ y $1,57 \mathrm{~cm} /$ año respectivamente. Las trayectorias de crecimiento fueron similares para los 10 árboles, donde los primeros tres años presentando un crecimiento similar, después cada árbol se va diferenciando, pero respetando su tendencia de crecimiento promedio.

Tabla 2. Control de calidad de las medidas del ancho de los anillos de crecimiento de la especie J. copaia.

Table 2. Quality control of the measurements of the width of the growth rings of the J. copaia species.

\begin{tabular}{|c|c|c|c|c|}
\hline \multirow{2}{*}{ Especie } & \multicolumn{2}{|c|}{$\mathrm{N}^{\circ}$ de árboles } & \multirow{2}{*}{$\begin{array}{c}\text { Cronología } \\
\text { (intervalo) }\end{array}$} & \multirow{2}{*}{$\begin{array}{l}\text { Edad } \\
\text { máxima }\end{array}$} \\
\hline & Antes & Después & & \\
\hline Jacaranda copaia & $10(40)$ & $10(34)$ & $1984-2018$ & 34 años \\
\hline
\end{tabular}

Tabla 3. Valores estadísticos de la datación y estandarización de la cronología de la especie J. copaia.

Table 3. Statistical values of the dating and standardization of the chronology of the J. copaia species.

\begin{tabular}{lc}
\hline Especie & Jacaranda copaia \\
\hline $\mathrm{N}^{\circ}$ de árboles & 10 \\
Intercorrelación (Promedio) & 0,361 \\
Sensibilidad (Promedio) & 0,364 \\
Cronología (Rango) & $1984-2018$ \\
Edad máxima & 34 años \\
\hline
\end{tabular}

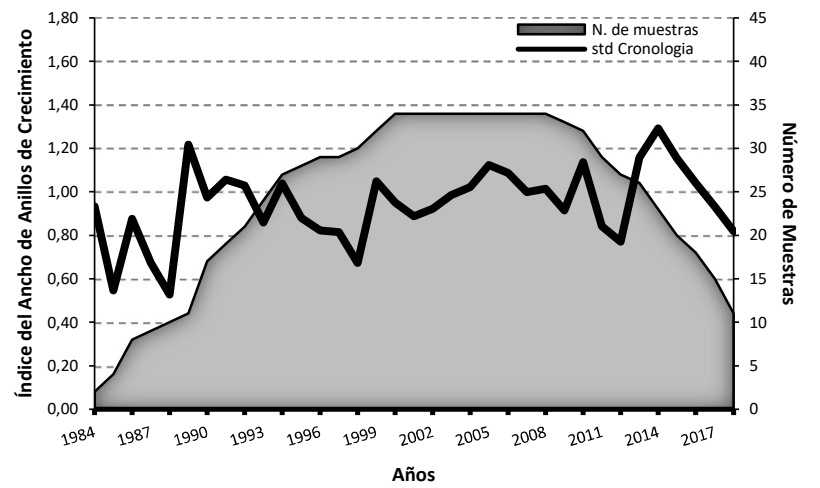

Figura 5. Cronología master de los índices del ancho de los anillos de crecimiento de la especie J. copaia.

Figure 5. Master chronology of the growth ring width indices of the J. copaia species.
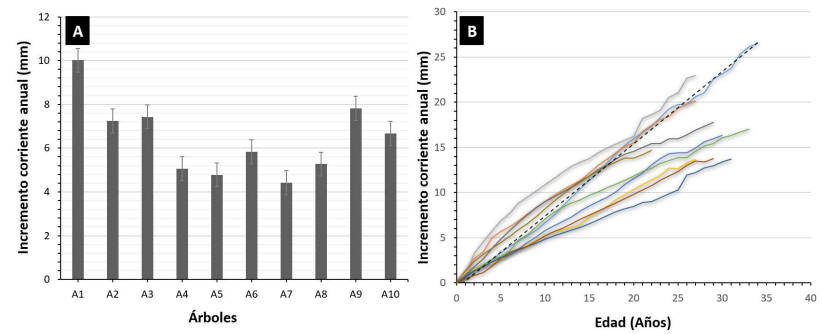

Figura 6. J. copaia ( $\mathrm{n}=10)$. A) Incremento corriente anual (ICA). B) Incremento en diámetro acumulado (IDA).

Figure 6. J. copaia $(\mathrm{n}=10)$. A) Annual current increase (ICA). B) Increase in accumulated diameter (IDA).

\subsection{El Fenómeno de El Niño y la cronología}

El crecimiento de los 10 árboles responde negativamente en los trimestres de diciembre, enero y febrero $(-0,05855)$, enero, febrero y marzo $(-0,06070)$, febrero, marzo y abril ($0,02546)$ y positivamente en los otros trimestres, teniendo una variación de $(0,03517$ a 0,18065$)$, sin embargo, ninguno de los trimestres llegó a ser significativo $(\mathrm{p}<0,05)$.

Se graficó la cronología obtenida de los 10 árboles de $J$. copaia, y los datos históricos del fenómeno de El Niño; en dicho gráfico se observa algunos episodios bien marcados, donde se observa una disminución del ancho de las camadas de crecimiento, en los años 1997, 2002 y 2009 (Figura 7), dicha reducción del ancho de las camadas de crecimiento, de J. copaia, muestra una disminución tasa de incremento en la población y puede estar conexa a los sucesos más severos del fenómeno de El Niño. El ancho de las camadas de crecimiento de J. copaia, fue correlacionado positivamente $(0,18065)$ con el índice del fenómeno El Niño Oceánico $(\mathrm{ONI})$, de noviembre, diciembre y enero. Se encontró una correlación positiva entre el fenómeno El Niño con la 
cronología de J. copaia de 0,18, lo que demuestra que influencia en su crecimiento.

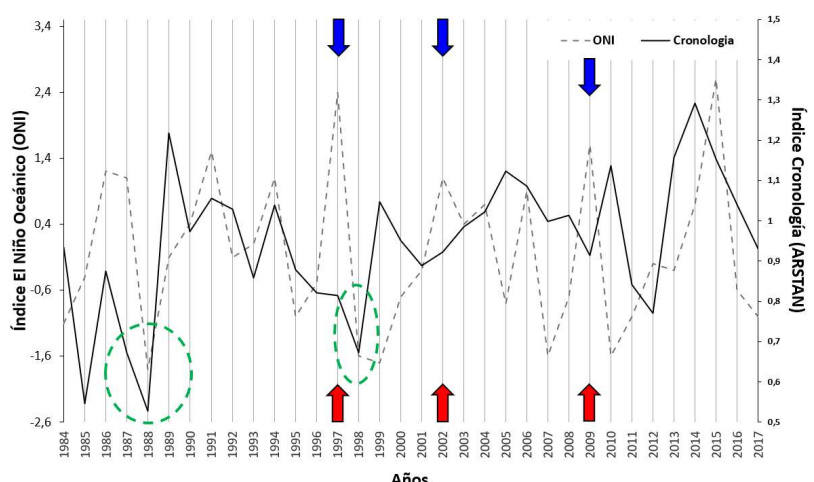

Figura 7. Relación entre los eventos de El Niño (línea trazada ceniza) y el ancho de los anillos de crecimiento de J. copaia (línea negra), con detalles de los anillos más cortos (flecha roja) y los de los eventos más severos de El Niño (flecha azul).

Figure 7. Relationship between El Niño events (ash line) and width of J. copaia growth rings (black line), with details of shorter rings (red arrow) and more severe El Niño events (blue arrow).

En la Figura 7, además se muestran dos episodios claramente distinguibles en los años 1988 y 1998; donde la cronología coincide exactamente con la curva ONI, y que si es para abajo representa el fenómeno de La Niña, con esto podemos inferir que cuando el fenómeno de La Niña fue significativo el crecimiento disminuyo posiblemente porque existió mucha precipitación lo que hizo que el suelo se saturo de agua, limitando su crecimiento para no sufrir embolia.

\section{DISCUSIÓN}

4.1. Caracterización de la sección transversal y de los anillos de Crecimiento

La descripción de la sección transversal de la madera de J. copaia, coincide con la literatura científica, sin embargo sobre la descripción de los anillos de crecimiento, describen que la especie presenta el límite de los anillos de crecimiento intermedios entre distintos e indistintos, y esta descripción también se encuentra en (IAWA, 1989), sin embargo, creemos que como la madera es de coloración clara y el anillo delimitado por zona fibrosa es distinto pero suavemente marcado (APARECIDO et al., 2019; LEÓN, 2007; PACE et al., 2015).

Por otro lado, en estudios realizados con diferentes especies del género Jacaranda, la anatomía de la madera de $J$. copaia, se distingue de otras especies del mismo género, por ejemplo: los vasos son de mayor diámetro y menos por milímetro cuadrado, los elementos del vaso y las fibras son más largos, y el número de células por línea de parénquima es mayor. Además, los rayos son menos por milímetro y más altos (DOS SANTOS; MILLER, 1997). Además, ALFARO et al. (2017) menciona que la especie J. copaia, exhibió los límites más distintos de los anillos de crecimiento de los árboles, que, combinado con su alta coherencia entre los árboles, sugiere que la incertidumbre es más baja en esta especie.

\subsection{Dendrocronología}

Desde fines de la década de los años ochenta, se conoce la anualidad de los anillos de crecimiento de la especie $J$. copaia, cuando DÉTIENNE (1989), estudio varias especies entre ellas J. copaia, en África tropical y Guyana Francesa, donde encontró que se delimitaban por zonas de fibras al comienzo de la madera temprana, además menciona que, los anillos perdidos o ausentes rara vez se registraron en estas especies. Finalmente concluye que la especie J. copaia, tiene potencial para la datación. Sin embargo, no se ha estudiado a fondo esta especie forestal nativa solo se menciona actualmente como especie de árbol de América tropical con anillos anules confirmados, cuando describen la familia botánica Bignoniaceae (SCHÖNGART et al., 2017). Además Jenkins (2009) , en su investigación demostró que la región de Madre de Dios presenta potencial para estudios dendrocronológicos, encontrando buenos resultados con las especies: Cedrela odorata, Ceiba pentandra, Hymenaea courbaril y menciona que existen especies prometedoras para estudios dendrocronológicos en la región como el caso de J. copaia. En esta investigación se confirma el potencial de dendrocronológico de J. copaia, una especie nativa de rápido crecimiento en los bosques tropicales de Perú, mostrando la anualidad de los anillos de crecimiento cruzando patrones de ancho de los anillos interanuales.

\subsection{Incremento radial de la especie}

En un estudio de crecimiento en diámetro de especies forestales en Guayana Venezolana, encontraron que el crecimiento anual de la especie J. copaia, presento en promedio $0,83 \mathrm{~cm} /$ año, estudiando parcelas permanentes de muestreo, este resultado esta similar a los encontrados en la presente investigación $(0,80 \mathrm{~cm} /$ año$)$, con técnicas dendrocronológicas, mostrando resultados próximos. En la investigación en Venezuela también se evaluaron otras especies de rápido crecimiento pioneras como Tachigali sp., de 0,80 cm/año; Cedropia spp., de 0,83 cm/año. Siendo estas especies de similar crecimiento que J. copaia (HERNÁNDEZ; CASTELLANOS, 2006).

Otro estudio realizado en Colombia sobre el crecimiento de árboles en un bosque pluvial tropical, evaluados por parcelas permanentes encontraron que las especies Myrciaria floribunda de $0,77 \mathrm{~cm} /$ año y Hyeronima antioquensis de 0,85 $\mathrm{cm} /$ año, que son cercanos a los encontrados en J. copaia (QUINTO; MORENO, 2010).

Estas investigaciones realizadas con las parcelas permanentes de muestreo coinciden con los valores de incremento anual similares a los encontrados con técnicas dendrocronológicas muestras la rapidez y la confiabilidad de los resultados a través de los anillos de crecimiento.

\subsection{El fenómeno de E1 Niño y la cronología}

ALFARO et al. (2017) menciona que fuertes eventos del fenómeno El Niño asociados a condiciones más secas y soleadas redujeron el crecimiento de los árboles tropicales, pero no su reproducción. Los efectos climáticos como el fenómeno de EL Niño pueden afectar en el crecimiento de las especies, pero puede diferir en la reproducción de estos. Importante señalar que esta conclusión fue realizada cuando realizo estudios de dendrocronología con la especie $J$. copaia. Además, ALFARO et al. (2017), manifiesta que sus análisis sugieren que el fenómeno El Niño tiene efectos negativos consistentes sobre el crecimiento radial en todas las especies de estudio incluyendo J. copaia. Y que estos efectos sobre el crecimiento radial y la reproducción fueron más pronunciados durante 1997-1998 El Niño. Además, SCHONGART et al. (2004), menciona que las últimas dos décadas han estado marcadas por un inusual y fuerte 
fenómeno de El Niño, como los eventos en 1982/1983, 1997/1998. Resultados que son reflejados en la presente investigación donde se observa que el año 1997 y 1998 el fenómeno El Niño fue altamente significativo con el pico alto y afecto considerablemente en el crecimiento de J. copaia, en la zona de estudio del fundo El Bosque en el Perú. Por último, estudios realizados en el norte de la región de Pantanal en el Brasil con la especie Vochysia divergens, mostraron que el fenómeno El Niño conducen a una disminución en la precipitación, lo que resulta en una disminución de los incrementos de diámetro de esta especie (FELFILI et al., 2018).

\section{CONCLUSIONES}

Confirmamos la anualidad de la formación de los anillos de crecimiento para la especie J. copaia, presenta potencial dendrocronológico en los bosques de Madre de Dios en el Perú; y mostramos el potencial dendrocronológico, evidenciado principalmente por la intercorrelación de sus anillos de crecimiento de 0,36 , sumado a la delimitación de los anillos de crecimiento.

El incremento anual de la especie J. copaia, confirma ser una especie de rápido crecimiento teniendo en promedio un crecimiento de $0,80 \mathrm{~cm} /$ año.

Los eventos extremos del fenómeno El Niño fue registrado en la cronología de J. copaia, asociada a épocas secas en los bosques de Madre de Dios y reduciendo su crecimiento.

Al comprobar que los anillos de crecimiento de la especie J. copaia, responden al fenómeno El Niño coloca a esta especie como potencial para estudios de dedroclimatología en los bosques tropicales de Madre de Dios y así entender más estos procesos.

Sugerimos que las futuras investigaciones puedan incluir rodajas de maderas para mejorar la cronología e incluir árboles de mayor diámetro para extender las cronologías de esta especie.

\section{AGRADECIMIENTOS}

Los autores agradecen a la Universidad Nacional Amazónica de Madre de Dios-UNAMAD, por las facilidades de sus laboratorios y del Fundo El Bosque, donde se realizaron las colectas de las muestras de madera. Al Programa Nacional de Becas y Crédito EducativoPRONABEC (Beca Presidente de la República).

\section{REFERÊNCIAS}

ALFARO-SÁNCHEZ，R.; MULLER-LANDAU，H. C.; WRIGHT, S. J.; CAMARERO, J. J. Growth and reproduction respond differently to climate in three Neotropical tree species. Oecologia, v. 184, n. 2, p. 531 541, 2017. DOI: 10.1007/s00442-017-3879-3.

ANDRADE, V. H.; MACHADO, S. do A.; FIGUEIREDO FILHO, A.; BOTOSSO, P. C.; MIRANDA, B. P.; SCHÖNGART, J. Growth models for two commercial tree species in upland forests of the Southern Brazilian Amazon. Forest Ecology and Management, v. 438, p. 215-223, 2019. DOI: 10.1016/j.foreco.2019.02.030.

APARECIDO, L. M. T.; SANTOS, J. dos; HIGUCHI, N.; KUNERT, N. Relevance of wood anatomy and size of Amazonian trees in the determination and allometry of sapwood area. Acta Amazonica, Manaus, v. 49, n. 1, p. 1-10, 2019. DOI: 10.1590/1809-4392201800961.

BELTRÁN, L. A.; VALENCIA, G. M. Anatomía de anillos de crecimiento de 80 especies arbóreas potenciales para estudios dendrocronológicos en la Selva Central, Perú. Revista de Biología Tropical, v. 61, n. 3, 2013. DOI: 10.15517/rbt.v61i3.11778.

BRIENEN, R. J. W.; HELLEC, G.; PONSD, T. L.; GUYOTE, J.-L.; GLOORA, M. Oxygen isotopes in tree rings are a good proxy for Amazon precipitation and El Nino-Southern Oscillation variability. Proceedings of the National Academy of Sciences, v. 109, n. 42, p. 16957-16962, 2012. DOI: 10.1073/pnas.1205977109.

BRIENEN, R. W.; ZUIDEMA, P. A. Relating tree growth to rainfall in Bolivian rain forests: a test for six species using tree ring analysis. Oecologia, v. 146, n. 1, p. 1-12, 2005. DOI: $10.1007 / \mathrm{s} 00442-005-0160-y$.

CALLADO, C.; SILVA NETO, S. da; SCARANO, F.; COSTA, C. Periodicity of growth rings in some floodprone trees of the Atlantic Rain Forest in Rio de Janeiro, Brazil. Trees, v. 15, n. 8, p. 492-497, 2001. DOI: 10.1007/s00468-001-0128-4.

DÉTIENNE, P. Appearance and Periodicity of Growth Rings in some Tropical Woods. IAWA Journal, v. 10, n. 2, p. 123-132, 1989. DOI: 10.1163/22941932-90000480.

DOS SANTOS, G.; MILLER, R. Wood Anatomy of Jacaranda (Bignoniaceae): Systematic Relationships in Sections Monolobos and Dilobos as Suggested by Twig and Stem Wood Rays. IAWA Journal, v. 18, n. 4, p. 369383, 1997. DOI: $10.1163 / 22941932-90001502$.

FELFILI, C. F.; CUNHA, C. N. da; ROSA, S. A.; PAIXÃO, E.; JUNK, W. J.; SCHÖNGART, J. Dendrochronological records of a pioneer tree species containing ENSO signal in the Pantanal, Brazil. Brazilian Journal of Botany, Reporter: Brazilian Journal of Botany, v. 41, n. 1, p. 167-174, 2018. DOI: 10.1007/s40415-017-0434-8.

FOSTER, R.; PARKER, T.; GENTRY, A. (Org.). The Tambopata-Candamo reserved zone of southeastern Perú: a biological assessment. Washington, DC: Conservation International, Department of Conservation Biology, 1994. (RAP working papers, 6).

GARCÍA, A.; CAMARERO, J.; ESPINOSA, C. Intra-annual stem increment patterns and climatic responses in five tree species from an Ecuadorian tropical dry forest. Trees, v. 31, n. 3, p. 1057-1067, 2017. DOI: 10.1007/s00468-017-1530-x.

HAYDEN, B. Annual growth rings in dry tropical forest trees. 2009. Library and Archives Canada = Bibliothèque et Archives Canada, Ottawa, 2009.

HERNÁNDEZ，L.; CASTELLANOS，H. Crecimiento diamétrico arbóreo en bosques de Sierra de Lema, Guayana venezolana: primeras evaluaciones. Interciencia, v. 31, n. 11, p. 787-793, 2006.

HOLMES, R. Computer-assisted quality control in tree-ring dating and measurement. Tree-ring bulletin, p. 11, 1983.

IAWA. IAWA list of microscopic features for hardwood identification. [s.1.]: IAWA Bull, 1989. v. 10.

KALNAY, E.; KANAMITSU, M.; KISTLER, R.; COLLINS, W.; DEAVEN, D.; GANDIN, L.; IREDELL, M.; SAHA, S.; WHITE, G.; WOOLLEN, J.; 
ZHU, Y.; CHELLIAH, M.; EBISUZAKI, W.; HIGGINS, W.; JANOWIAK, J.; MO, K. C.; ROPELEWSKI, C.; WANG, J. LEETMAA, A.; REYNOLDS, R.; JENNE, R.; JOSEPH, D. The NCEP/NCAR 40-Year Reanalysis Project. Bulletin of the American Meteorological Society, v. 77, n. 3, p. 437-471, mar. 1996. DOI: 10.1175/15200477(1996)077<0437:TNYRP>2.0.CO;2.

LEÓN, W. Anatomía del Xilema secundario de diez especies de la familia Bignoniaceae de Venezuela. Acta Botánica Venezuelica, v. 30, n. 2, p. 361-384, 2007.

LISI, C. S.; TOMAZELLO, M.; BOTOSSO, P. C.; ROIG, F. A.; MARIA, V. R. B.; FERREIRA-FEDELE, L.; VOIGTH, A. R. A. Tree-ring formation, radial increment periodicity, and phenology of tree species from a seasonal semi-deciduous forest in southeast Brazil. Iawa Journal, v. 29, n. 2, p. 189-207, 2008.

MALHI, Y.; PHILLIPS, O. L.; LLOYD, J.; BAKER, T.; WRIGHT, J.; ALMEIDA, S.; ARROYO, L.; FREDERIKSEN, T.; GRACE, J.; HIGUCHI, N.; KILLEEN, T.; LAURANCE, W. F.; LEAÑO, C.; LEWIS, S.; MEIR, P.; MONTEAGUDO, A.; NEILL, D.; NÚÑEZ VARGAS, P.; PANFIL, S. N.; PATIÑO, S.; PITMAN, N.; QUESADA, C. A.; RUDAS-LL., A.; SALOMÃO, R.; SALESKA, S.; SILVA, N.; SILVEIRA, M.; SOMBROEK, W. G.; VALENCIA, R.; VÁSQUEZ MARTÍNEZ, R.; VIEIRA, I. C G.; VINCETI, B. An international network to monitor the structure, composition and dynamics of Amazonian forests (RAINFOR). Journal of Vegetation Science, v. 13, n. 3, p. 439-450, 2002. DOI: 10.1658/1100-9233(2002)013[0439:AINTMT]2.0.CO;2.

MENDIVELSO, H. A.; CAMARERO, J. J.; GUTIÉRREZ, E.; ZUIDEMA, P. A. Time-dependent effects of climate and drought on tree growth in a Neotropical dry forest: Short-term tolerance vs. long-term sensitivity. Agricultural and Forest Meteorology, v. 188, p. 13-23, 2014. DOI: 10.1016/j.agrformet.2013.12.010.

MENDIVELSO, H. A.; CAMARERO, J. J.; GUTIÉRREZ, E. Dendrochronology in Neotropical dry forests: methods, advances and applications. Ecosistemas, Reporter: Ecosistemas, v. 25, n. 2, p. 66-75, 2016. DOI: 10.7818/ECOS.2016.25-2.08.

NOAA. NOAA's Climate Prediction Center. 2017. Disponível em: http://origin.cpc.ncep.noaa.gov/products/analysis_mo nitoring/ensostuff/ONI_v5.php. Acesso em: 30 mar. 2018.

PACE, M. R.; LOHMANN, L. G.; OLMSTEAD, R. G.; ANGYALOSSY, V. Wood anatomy of major Bignoniaceae clades. Plant Systematics and Evolution, v. 301, n. 3, p. 967-995, mar. 2015. DOI: 10.1007/s00606-014-1129-2.
PORTAL, L. Potencial Dendrocronológico de Árvores da Família Fabaceae na Floresta Tropical Amazônica do Peru. 2017. 149 f. Universidade Federal Rural do Rio de Janeiro, Rio de Janeiro - Brasil, 2017. Disponível em: https://tede.ufrrj.br/jspui/bitstream/jspui/2060/2/201 $7 \% 20-$

\%20Leif\%20Armando\%20Portal\%20Cahuana.pdf.

QUINTO, H.; MORENO, F. Crecimiento de árboles en un bosque pluvial tropical del chocó y sus posibles efectos sobre las líneas de energía. Revista de Biologia e Ciências da Terra, v. 10, n. 2, p. 12-28, 2010.

SCHÖNGART, J. et al. Dendroecological Studies in the Neotropics: History, Status and Future Challenges. In: AMOROSO, M. et al. (org.). Dendroecology. Cham: Springer International Publishing, 2017. v. 231. p. 35-73. DOI: 10.1007/978-3-319-61669-8_3. Disponível em: http:/ /link.springer.com/10.1007/978-3-319-616698_3. Acesso em: 16 mar. 2020

SCHONGART, J. J.; PIEDADE, W. J.; AYRES, M. T. F.; HÜTTERMANN, J. M.; WOERBES, M. A. Teleconnection between tree growth in the Amazonian floodplains and the El Nino-Southern Oscillation effect. Global Change Biology, Reporter: Global Change Biology, v. 10, n. 5, p. 683-692, 2004. DOI: 10.1111/j.1529-8817.2003.00754.x.

TIMMERMANN, A.; OBERHUBER, J.; BACHER, A.; ESCH, M.; LATIF, M.; ROECKNER, E. Increased El Niño frequency in a climate model forced by future greenhouse warming. Nature, v. 398, n. 6729, p. 694-697, 1999. DOI: 10.1038/19505

VIEIRA, J. R.; GROENENDIJK, P.; LISI, C. S. Dendrochronological potential of four neotropical dryforest tree species: Climate-growth correlations in northeast Brazil. Dendrochronologia, Reporter: Dendrochronologia, v. 53, p. 5-16, 2019. DOI: 10.1016/j.dendro.2018.10.011.

VOLLAND，F. V.; BRÄUNING，A.; GANZHI，O.; PETERS, T.; MAZA, H. Radial stem variations of Tabebuia chrysantha (Bignoniaceae) in different tropical forest ecosystems of southern Ecuador. Trees, v. 25, n. 1, p. 39-48, 2011. DOI: 10.1007/s00468-010-0461-6.

WORBES, M.; RASCHKE, N. Carbon allocation in a Costa Rican dry forest derived from tree ring analysis. Dendrochronologia, v. 30, n. 3, p. 231-238, 2012. DOI: 10.1016/j.dendro.2011.11.001. 